\title{
Effect of Traditional Chinese Medicine Collaborative Model (TCMCM) combined Adjuvant Chemotherapy in IIIb and IIIC Gastric Cancer: Protocol for a Randomized Controlled Trial
}

zhaoyan li

Longhua hospital, Shanghai University of Traditional Chinese Medicine

Jia Li

Longhua hospital,Shanghai unversity of Traditional Chinese Medicine

Nida Cao

Longhua hospital, Shanghai University of Traditional Chinese Medicine

Xiaohong Zhu

Longhua Hospital,Shanghai University of Traditional Chinese Medicine

Yan Xu

Longhua Hospital,Shanghai University of Traditional Chinese Medicine

\section{Chen Han}

Longhua Hospital,Shanghai University of Traditional Chinese Medicine

\section{Mengmeng Qin}

Shanghai University of Traditional Chinese Medicine

Feng Liu

Longhua Hospital,Shanghai University of Traditional Chinese Medicine

\section{Guangtao Zhang}

Longhua Hospital, Shanghai University of Traditional Chinese Medicine

\section{Rui Wang}

Longhua Hospital,Shanghai University of Traditional Chinese Medicine

\section{Xiang Xia}

Renji Hospital,Shanghai Jiaotong University School of Medicine

\section{Gang Zhao}

Shanghai Jiaotong University School of Medicine Xinhua Hospital

\section{Xiangkun Huan}

Jiangsu Province Hospital of Chinese Medicine,Nanjing University of Chinese Medicine

\section{Jin Fan}

Fundan Unversity Shanghai Cancer Center

Aiguang Zhao ( $\nabla$ aiguangzhao@qq.com )

Shanghai University of Traditional Chinese Medicine

\section{Study protocol}

Keywords: gastric cancer, traditional Chinese medicine collaborative model, adjuvant chemotherapy, DFS, Randomized controlled trial

Posted Date: June 28th, 2021

DOl: https://doi.org/10.21203/rs.3.rs-486842/v1 
License: (c) (i) This work is licensed under a Creative Commons Attribution 4.0 International License. Read Full License

Version of Record: A version of this preprint was published at Trials on January 21st, 2022. See the published version at https://doi.org/10.1186/s13063-022-06013-5. 


\section{Abstract}

\section{Background}

Metastasis and/or recurrence are the primary cause in decreasing the survival time of gastric cancer patients who experienced radical operation. Among whom, patients with stage IIIb and IIIc are especially in high risk of metastasis and recurrence, result in a significant poor survival time than patients with earlier stages. Herbal medicines are natural substances that have been used

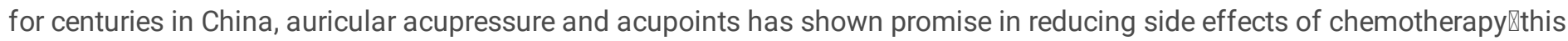

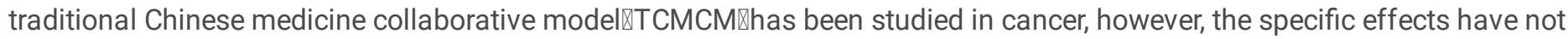
been systematically evaluated. This study was designed to evaluate whether TCMCM can decrease adverse effects after chemotherapy and reduce the recurrence rate and metastasis in stage IIIb and IIIc gastric cancer.

Method/design

This prospective $₫$ multicenter, randomized, open-label trail will recruit 260 patients with stage IIIb and IIlc gastric cancer who undergo radical surgery with D2 lymphadenectomy. Randomization to usual adjuvant chemotherapy or the intervention (TCMCM) with a 1:1 ratio will be used. Patients in the intervention group received an oral traditional Chinese formula, auricular acupressure and acupoints, all participants will be continuing to receive usual adjuvant chemotherapy. The primary outcome is 3year disease free survival rate. Secondary outcomes include quality of life『side effects caused by chemotherapy and safety

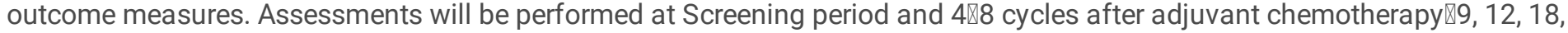
24,30 and 36 months after randomization, and adverse events will be recorded.In addition,biological samples will be collected for mechanism exploration studies.

Discussion

This will be the first clinical trial to evaluate the disease-free survival (DFS) and improvements in quality of life in patients of stage IIIb and IIIc gastric cancer receiving TCMCM, which may be used to formulate a standardized TCMCM plan. We are also performing this trial to assess the feasibility of a larger-scale clinical trial in the future.

Trail registration

ClinicalTrials.gov『NCT03607656. Registered on 1 July 2018.The final protocol version was V1.1.

\section{Background}

Gastric cancer is one of the most common malignancy and the second leading cause of cancer-related death thought the world[1]. More than half of the world's gastric cancer cases occur in Asia, accounting for $42 \%$ of China[2, 3]. Due to lack of effective diagnostic and therapeutic strategies, more than $80 \%$ gastric cancer patients in China are already in advanced stages at the time of diagnosis and the 5-year survival rate remains quite low[4]. Among whom, patients with stage IIIb and IIIc are especially in high risk of metastasis and recurrence, result in a significant poor survival time than patients with earlier stages.

With the continuous improvement of postoperative chemotherapy regimens, the DFS and overall survival (OS) of gastric cancer were prolonged[5, 6], Several clinical trials have established the effects for adjuvant chemotherapy in the treatment of gastric cancer[7] [8] [9]. Chemotherapy has been the standard treatment for patients with advanced gastric cancer[10]. However, about $40 \%-60 \%$ of patients with gastric cancer after resection have recurrence or metastasis, the prognosis is still poor[11], and they are toxic and often cause side effects affecting the quality of life of patients[12]. Therefore, there is still an urgent need to explore new methods to improve the prognosis of patients after radical gastrectomy.

In China $\triangle$ The effect of herbal medicines have been demonstrated material and beneficial effects in improving available cancer therapeutics[13,14], auricular acupressure and acupoints are the nonpharmacological and noninvasive therapy that can diminish the side effects of chemotherapy and improve the quality of life囚they are safe囚effective and easily accepted by patients[15, 16]. Hence, many patients diagnosed with cancer always choose to resort to TCM therapy. However, due to the individual treatment model of TCM and the need for frequent adjustments, it is difficult to design rigorous modern controlled trials for TCMCM, large-

Page $3 / 12$ 
scale, randomized-controlled trials evaluating the efficacy of TCMCM \and especially high-quality designed clinical trials are lacking, and only a few studies have comprehensively evaluated the prognostic value of this treatment in patients of GC[17]. We will assess the clinical effectiveness, quality of life, and safety outcomes to evaluate the efficacy of TCMCM in IIIb and IIIc gastric Cancer, we designed this randomized, open-label study.

\section{Methods/design}

\section{Study design}

This study protocol was registered in the ClinicalTrials Registry \https://register.clinicaltrials.gov/『with the number NCT03607656 on 1 July 2018.The study will identify patients with stage IIlb and Illc gastric cancer who undergo curative D2 gastrostomy beginning in June 2018囚at three centers in China, including Longhua Hospital affiliated to Shanghai University of TCM, Renji Hospital affiliated to Shanghai Jiaotong University School of Medicine and Jiangsu Province Hospital of Chinese Medicine affiliated to Nanjing University of Chinese Medicine. The present study is designed as a longitudinal randomized interventional study with two study arms. Patients in the intervention group received an oral traditional Chinese formula, auricular acupressure and acupoints, all participants will be continuing to receive usual adjuvant chemotherapy which according to the National Comprehensive Cancer Network (NCCN) gastric cancer Treatment Guidelines. The trial design and checklist for this study protocol are summarized in Fig. 1 and Additional file 1 respectively.

\section{Sample size estimate}

Our previous study described the 3-year disease-free survival rate was $49 \%$ who underwent the Chinese herbal medicines combined with chemotherapy and $28 \%$ in the chemotherapy group. With a power of 0.9 , the total number of randomized controlled trials was 197 . Considering the $20 \%$ of follow-up loss, the confinement factor in different hospitals increased by $10 \%$ of the sample size, we will recruit a total of 260 patients for this trial.

\section{Recruitment}

Before the research, investigators of three centers are responsible for the introduction the clinical research related matters and the records of the baseline data respectively, and prospective participants will be informed of the benefit and possible risk associated with this study. Participants will be told that they can withdraw from the trial at any time without specifying reasons. Eligible participants will be randomized into two groups with different treatments once informed consent has been obtained. The investigator must have the expertise, qualification and ability of the clinical trial. After the qualification examination, the personnel requirements are relatively fixed. Every investigator will participate in training on the fully understand the specific connotation of the clinical study programme.

\section{Criteria:}

\section{Inclusion Criteria:}

- Histologically proven gastric carcinoma with radical operation, TNM (primary tumor, regional nodes, metastasis) stage IIIb or IIIc (The 8th edition of the tumor-node-metastasis (TNM) staging system from the American Joint Cancer Committee/Union Internationale Contre le Cancer (AJCC/UICC));

-The age of onset is 18 years old;

- Karnofsky performance status higher than 70;

- Adequate hepatic, renal, cardio and hematologic function;

- With patients' consent and comply to long term follow-up.

\section{Exclusion Criteria:}

Page $4 / 12$ 
- Gastrectomy beyond D2, or TNM stage beyond IIIb and IIIc;

- Histological type beyond gastric carcinoma;

- Conversion chemotherapy before surgery;

- Concurrent cancer;

- Women of gravid or lactating; patients with mental illness;

- Uncontrolled significant comorbid conditions\such as unstable angina or myocardial infarction.

\section{Randomization}

In order to minimize selection bias, all eligible patients will be randomly assigned in a 1:1 ratio to the treatment group or the control group. Randomization will be performed by a professional, independent statistician who is not involved in the recruitment process of the study. The central randomization software "Clinstat Group" APP version 1.2.0. will be used for group allocation according to a randomization list. Randomization has two stratification factors, the first one is disease stage of IIIb or IIIc, and the second one is center (Longhua hospital, Renji hospital or Jiangsu Province Hospital of Chinese Medicine). The software server side pre-establishes two independent random lists according to the stratification factor (stage: IIIB period vs. IIIC period), and each center shares a random list. Concealed allocation is guaranteed by the randomization program. Once the inclusion criteria have been fulfilled and informed consent has been received, clinical researcher of centers will tell the statistician the information of patients 'gender, age, and the stage『and then the statistician will log on the Clinstat Group to enter this basic information. Then the result is immediately presented on the screen. Neither investigators nor patients were masked to treatment assignment in this open-label trial.

\section{Intervention}

Enrolled patients in the control group will be accepted the treatment of adjuvant chemotherapy $\otimes T$ The chemotherapy can choose intravenous oxaliplatin $130 \mathrm{mg} / \mathrm{m}(2)$ on day 1 plus oral capecitabine $1000 \mathrm{mg} / \mathrm{m}(2)$ twice daily on days 1-14, every 21 days. Two additional chemotherapy regimens added after 27 June 2019 .intravenous oxaliplatin $100 \mathrm{mg} / \mathrm{m}(2)$ on day 1 plus oral S-1 $40 \mathrm{mg} / \mathrm{m}(2)$ twice daily on days $1-14$, every 21 days; or intravenous docetaxel $40 \mathrm{mg} / \mathrm{m}(2)$ on day 1 plus oral S-1 40mg/m(2) twice daily on days $1-14$,every 21 days; or intravenous docetaxel $50 \mathrm{mg} / \mathrm{m}(2)$ on day 1 plus intravenous oxaliplatin $85 \mathrm{mg} / \mathrm{m}(2)$ on day 1 plus 5-FU intravenously CIV24h on day 1, every 14 days; Each participant should take eight (8) cycles of chemotherapy. The choice of chemotherapy regimen, including adjustments, are directed by one of two Chief physicians.

In addition to the above-mentioned adjuvant chemotherapy treatment, participants in the treatment group will receive an oral traditional Chinese formula based on the Jianpi theory, The major herbal formula components were Radix Pseudostellariae (12g), Rhizoma Atractylodis Macrocephalae (12g), Poria(30g), Rhizome Pinelliae Preparata (9g), green tangerine peel (4.5g), Concha Ostreae (30g), and Prunella vulgaris $(9 \mathrm{~g})$. The herbal components above were mainly provided by Sunbow Pharmaceutical (7600 Zhongchun Road, Shanghai 201100, China). This Chinese medicine factory has quality control (certified GMP ShanghaiG0172). The preparation of the formula water decoction and its quality control had been described in our previously published articles. The patient started taking the Chinese herbal within 8-12 weeks after enrollment. The administration method was mainly oral administration of the decoction, and one dose per day was taken in 2 to 4 times. The clinical interventions of oral traditional Chinese formula were designed and adjusted on by senior TCM physicians in every center, and the formula is based on syndrome differentiation by examination, listening, smelling, interrogation, pulse-taking and palpation, the dose of each ingredient in the prescription will be decided by the physician. According to the clinical syndrome differentiation, adjust the medication every $1 \sim 2$ weeks, continuous treatment for 6 months and above or until disease progresses (relapse/metastasis/die), unacceptable toxicity, or withdrawal of consent.

On the first day of every cycle adjuvant chemotherapy, participants received auricular acupressure and acupoints by the research nurse. Auricular acupressure and acupoints points were chosen by a panel of expert faculty from Shanghai university of TCM. The auricular acupressure selected for this study were shenmen囚jiaogan and stomach. Magnetic beads held in place by tape 
were used to stimulate the three auricular acupoints, one side of the ear was selected for each time, and both sides of the ear were used in turn. Three times per day for $3 \mathrm{~min}$ per time ( $9 \mathrm{~min}$ total】. The acupoint selected for this study were neiguanखzusanli and fenglong, The acupoint remained on the skin points for 24 hours. Even if they did not experience uncomfortable.

\section{Biological specimens}

Next Generation Sequencing (NGS) technology is used to observe the mutations of tumorigenesis-related genes and potential treatment-related genes in some patients, and to assess the relationship between tumor mutation burden (TMB) and the prognosis of gastric cancer. Blood was collected 3 times before surgery, after chemotherapy, regeneration and metastasis. Patients without recurrence/metastasis will be examined 24 months after enrollment or at the end of the study.

\section{Study endpoints}

\section{Primary endpoint}

The primary endpoint is 3-year disease free survival rateXDFS was defined as the date of randomization until the date of progression(tumor metastasis or recurrence) or the date of death from any cause. If symptoms or signs of suspected recurrence or metastasis or abnormal tumor markers are found during follow-up, CT/MRI/gastroscopy should be performed promptly and PET-CT should be performed if necessary. All subjects Used the response evaluation criteria in solid tumors version 1.1(RECIST 1.1) to estimate the objective curative effect. If workup revealed relevant tumor markers persisted abnormally, However CT or MRI examinations failed to reveal any mass, it was evaluated by the research team's expert group. Kaplan Meier curves will be

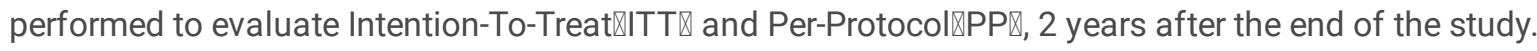

\section{Secondary Outcome Measure}

\section{EORTC QLQ-C30 scale}

European Organization for Research on Treatment of Cancer Quality of Life Questionnaire-Core 30هEORTC QLQ-C30『showed acceptable reliability in assessing the quality of life of cancer patients, this questionnaire focuses on physical condition and the quality of life of all participants. All results are recorded in the CRF table and the efficacy is assessed using scoring method. Assessments will be performed at Screening period and 4, 8 cycles after adjuvant chemotherapy, 9, 12, 18, 24, 30 and 36 months after randomization.

\section{Side effects of chemotherapy}

Side effects that occur during chemotherapy should be documented. Using NCI-CTC standard to record adverse effects of chemotherapy and treatments. compare toxicity and adverse events according to the NCl-CTC anti-cancer drugs in acute and subacute toxicity criteria and the Levi special sensory nerve toxicity grading criteria for oxaliplatin. Clinical researchers adjust medications based on toxic side effects. After the completion of treatment, follow-up examination was conducted along with the imaging examination. When a potentially dangerous or life-threatening adverse reaction occurs, a reduction in the dose or interruption of the drug can be allowed.

\section{Safety outcomes}

Safety indicators included assessment of vital signs at each visit, weight, KPS score, blood, liver and kidney function, fecal routine, and electrocardiogram. Adverse events (AEs) were defined as all unexpected or unintended symptoms, signs, or disorders that developed to patients who received adjuvant chemotherapy, and no regard was given to their causality with chemotherapy. According to the $\mathrm{NCl}$ common adverse events evaluation standard (v4.02) to compare adverse events.If serious adverse events occur during the study period, participants can withdraw from the study and the patient needs to complete the examinations and evaluations as much as possible.

\section{Follow-up}


Follow-up appointments for all patients will take place at Screening period and 4,8 cycles after adjuvant chemotherapy $₫ 9,12,18$, 24,30 and 36 months after randomization. Tumor marker examinations are checked every 3 months for the first two years, then every six months. A full abdominal augmentation CT/MRI and chest CT examination were performed every 3 months in a year, and then every six months; the gastroscope was examined once a year. All patients are followed up for 3 years or until recurrence, metastasis or death.

\section{Withdrawal of patients}

Patients can discontinue their participation in the study at any time for any reason if they wish to do so without any consequences. further, study medication can be stopped prematurely in case of serious adverse reaction, or if, in the investigator's opinion, study medication should be stopped for any other reason. If the patients in the control group continued to take Chinese herbal medicine orally in other hospitals for 6 months, they were judged to be out of the group.

\section{Data collection and management}

The investigators and research coordinator will be required to study the researcher's manual carefully before the trail begins. Medical records are original records and cannot be changed. The original record must not be altered when making any corrections, and can only be accompanied by an explanation and date, signed by the physician participating in the clinical trial. All patient data collected during this trial will be managed in using an electronic case report form (eCRF). The management team will review for quality and audit the eCRFs, and queries will be sent out to each participating center regularly. Data monitoring will also be conducted by way of site visits. The data will be managed and analyzed according to the study protocol. After each topic is tested and reviewed by the auditor, the data administrator locks the data until the last topic data is locked. Data Monitoring Committee $\varangle \mathrm{D} M C \varangle$ will be carried out by Xiyuan Hospital of China Academy of Chinese Medical Sciences. The data management are not involved in clinical observations.

\section{Statistical analysis}

SPSS Statistics version 22.0 will be the primary tool for data management and statistical analyses. All patients will be followed according to the protocol, and both intention-to-treat and per protocol analyses will be performed. Missing data will be adjusted for using the last observation information to obtain a complete database. Besides descriptive statistics and visualizations, Independent sample T test and Chi-square test (X2test) will be used for numerical variables and categorical variables respectively in the comparison between the two groups. When the distribution of variables is abnormal, a non-parametric test will be selected. Univariate analysis and survival rate calculations were performed using the Kaplan-Meier method, and the Log-rank test was used to compare the factors. The association of DFS with each clinical/demographic factor was compared between the two groups using the Kaplan-Meier analysis. Multivariate data are analyzed using COX regression analysis, trend testing, and so on. Statistical analysis of the research materials will be carried out by Fudan University Cancer Hospital. Statistical analysts are not involved in clinical observations.

\section{Table 1}

Page $7 / 12$ 


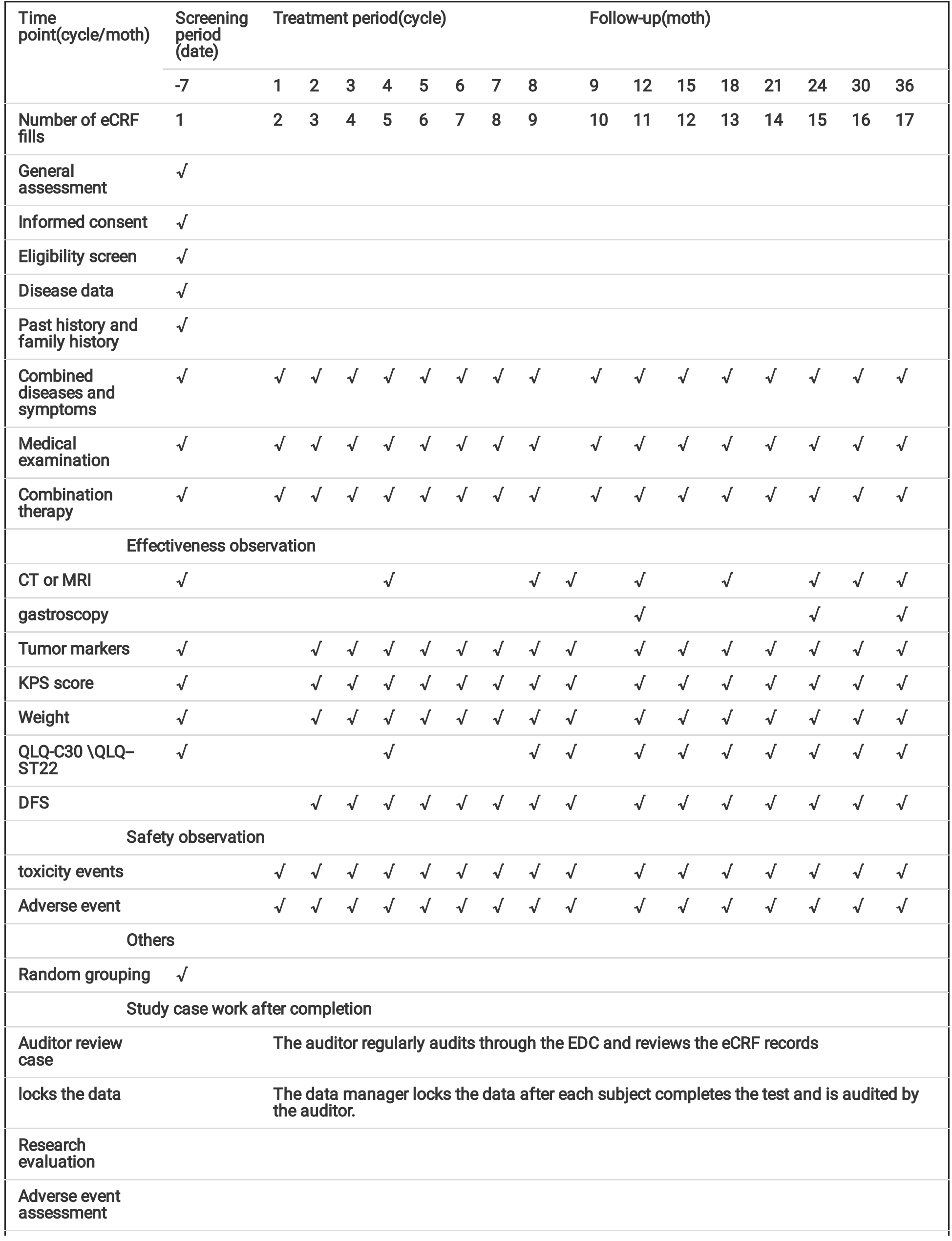




\section{Discussion}

Reports from a number of clinical studies demonstrated that TCM can improve the quality of life邓reduce Side effects[18]and prolong overall survival[19,20].TCM treatment include herbal medicine, acupuncture, acupoints and others. Herbal formula we chosen in this study is a primitive Chinese herbal formula designed in the 1980s. Our previous clinical study showed that it can prolong the OS and DFS of advanced gastric cancer [21,22]. Although the results show that it is effective, these studies are observational studies and non-randomized observational methods would carry a high risk of bias. Due to the special nature of traditional Chinese medicine, such as individualization, dynamic variability and medical experience, the study chooses to use randomized controlled trials without blind methods to strictly control the various biases that may occur in the implementation of clinical trials. It aims to balance the confounding factors and objectively evaluate the value of TCMCM intervention in the comprehensive treatment of patients with stage IIIB and IIIC gastric cancer. in our study, Auricular and acupoint application methods are the same for patients in the intervention group.Furthermore,free acupuncture and acupoints treatment will becomes more acceptable and conducive to reducing the dropout rate. In addition, our team will provide one-to-one health guidance to each patient. Therefore, we believe that most patients should be able to cooperate with the treatment and ensure good adherence to the program during the 2-year follow-up period.

In our randomized clinical trial, we tried to assess not only disease-free survival but also quality of life, side effects and Safety indicators. Side effects of chemotherapy affect prognoses in patients with gastric cancer and therefore need to be assessed, treated, and prevented. However, neither preventive nor therapeutic methods have been established for these therapies. The present study is the first multicenter, randomized, open-label study conducted to clarify the effects of TCMCM, for the prevention and treatment of survival prognosis and quality of life in patients with stage IIIB, IIIC gastric cancer after surgery. After the completion of this study, we expect our trial can not only preliminarily evidence about the efficacy of the Chinese medicine, but also provide a more standardized and humanized treatment plan for the postoperative patients with gastric cancer, which is beneficial to more and more patients.

\section{Trial status}

Protocol version: 4 Trial registration: ClinicalTrials.gov Registration number: NCT03607656

https://register.clinicaltrials.gov/NCT03538678) Date of trial registration: 1 July 2018

Was this trial prospectively registered? No

Date recruitment began: June 2018. Recruiting is ongoing

Anticipated completion date: December 2021.

\section{Abbreviations}

TCHM:Traditional Chinese Herbal Medicine; CT: Randomized control trial;DFS:disease free survival; AJCC/UICC囚the American

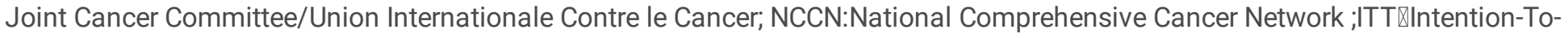
Treat\PP囚Per-Protocol\EORTC QLQ-C30:European Organization for Research and Treatment of Cancer (EORTC) Gastric-Cancer-

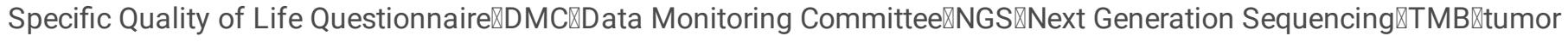
mutation burden.

\section{Declarations}




\section{Acknowledgements}

We gratefully acknowledge all the participants enrolled in this trial.

\section{Funding}

The research was supported by the Ministry of Science and Technology National Key Research and Development Program (2017YFC1700605).Funds are used for personnel,material and testing. None of the funding sources had any role in design of the study, nor will they be involved in the collection, analysis, interpretation of data and in writing the manuscript.

\section{Availability of data and materials}

The datasets used during the current study are available from the corresponding author on reasonable request.

\section{Authors contributions}

$A G Z, Y X, Z Y L$ and $G Z$ are responsible for the design of the trial with $A G Z$ serving as the primary investigator. $Z Y L, J L, N D C, X H Z$, $Y X, C H, M M Q, F L, R W, G T Z, X X, X K H$ are responsible for the collection of the data and the implementation of the protocol. ZYL and JF are responsible for the design of the smartphone app. ZYL and JL drafted the manuscript. All authors are responsible for drafting this document. All authors read and approved the final manuscript.

\section{Ethics approval and consent to participate}

Before the implementation of the study, the protocol of this study has been approved by the Ethics Committees of Longhua

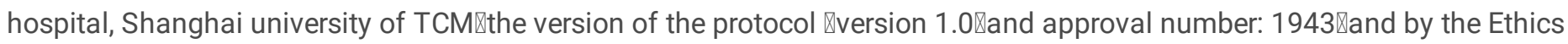
Committee of two other participating hospitals. Participants in the trail will provide written consent prior to participation. In 27 June $2019 \bigotimes$ the chemotherapy regimen of Xelox and FLOT added into the protocol by the principal investigator with the approval of the ethics committees of Longhua hospital囚Shanghai university of TCM.

\section{Consent for publication}

Not applicable

\section{Competing interests}

All authors declare that there is no conflict of interest.

\section{References}

1. Chen W, Zheng R, Baade PD, Zhang S, Zeng H, Bray F, et al.Cancer statistics in China, 2015. Ca Cancer J Clin.2016; 66(2):115-32.

2. Ferlay J, Soerjomataram I, Dikshit R, Eser S, Mathers C, Rebelo M, et al.Cancer incidence and mortality worldwide: sources, methods and major patterns in GLOBOCAN 2012. Int J Cancer.2015;136(5):E359-86.

3. Wei W, Zhe S, Jing-Yu D, Xiao-Long Q, Xing-Yu F, Cheng F, et al.A novel nomogram individually predicting disease-specific survival after D2 gastrectomy for advanced gastric cancer. Cancer Communications.2018;38(1):23.

4. Higgins RS, Brown RN, Chang PP, Starling RC, Ewald GA, Tallaj JA, et al.A multi-institutional study of malignancies after heart transplantation and a comparison with the general United States population. J Heart Lung Transplant, 2014;33(5):478-85.

5. Foo M, Leong T.Adjuvant therapy for gastric cancer: current and future directions. World Journal of Gastroenterology $.2014 ; 20(38): 13718-27$. 
6. Takagi W, Terashima M.Adjuvant Chemotherapy for Gastric Cancer. Gan to kagaku ryoho Cancer \& chemotherapy. 2016;43(2):151-5.

7. Sasako M, Sakuramoto S, Katai H, Kinoshita T, Furukawa H, Yamaguchi T, et al.Five-year outcomes of a randomized phase III trial comparing adjuvant chemotherapy with S-1 versus surgery alone in stage II or III gastric cancer. Journal of clinical oncology : official journal of the American Society of Clinical Oncology.2011;29(33):4387-93.

8. Noh SH, Park SR, Yang HK, Chung HC, Chung IJ, Kim SW, et al: Adjuvant capecitabine plus oxaliplatin for gastric cancer after D2 gastrectomy (CLASSIC): 5-year follow-up of an open-label, randomised phase 3 trial. The Lancet Oncology.2014;15(12):1389-96.

9. Al-Batran SE, Homann N, Pauligk C, Goetze TO, Meiler J, Kasper S, et al: Perioperative chemotherapy with fluorouracil plus leucovorin, oxaliplatin, and docetaxel versus fluorouracil or capecitabine plus cisplatin and epirubicin for locally advanced, resectable gastric or gastro-oesophageal junction adenocarcinoma (FLOT4): a randomised, phase 2/3 trial. Lancet (London, England) .2019;393(10184):1948-57.

10. Smalley SR, Benedetti JK, Haller DG, HundahI SA, Estes NC, Ajani JA, et al.Updated Analysis of SWOG-Directed Intergroup Study 0116: A Phase III Trial of Adjuvant Radiochemotherapy Versus Observation After Curative Gastric Cancer Resection. Journal of Clinical Oncology Official Journal of the American Society of Clinical Oncology.2012;30(19):2327-33.

11. Schmidinger M.Postoperative chemotherapy for gastric cancer. The oncologist.2006; 11(2):136.

12. Schwartzberg LS, Modiano MR, Rapoport BL, Chasen MR, Gridelli C, Urban L, et al.Safety and efficacy of rolapitant for prevention of chemotherapy-induced nausea and vomiting after administration of moderately emetogenic chemotherapy or anthracycline and cyclophosphamide regimens in patients with cancer: two randomised, active-controlled,double-blind,phase 3 trials. 2015;16(9):1079-89.

13. Efferth T, Li PCH, Konkimalla VSB, Kaina B.From traditional Chinese medicine to rational cancer therapy. Trends in Molecular Medicine.2007;13(8):353-61.

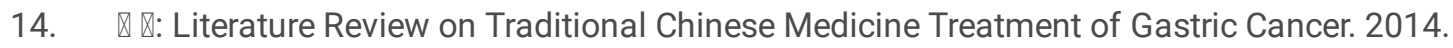

15. Huang Z, Zongshi Q, Qin Y, Yuanxuan W, Zhishun L.Moxibustion for Chemotherapy-Induced Nausea and Vomiting: A Systematic Review and Meta-Analysis. Evidence-Based Complementary and Alternative Medicine.2017;(2017):1-11.

16. Eghbali M, Yekaninejad MS, Varaei S, Jalalinia SF, Samimi MA, Sa'Atchi K.The effect of auricular acupressure on nausea and vomiting caused by chemotherapy among breast cancer patients. Complement Ther Clin Pract.2016;24:189-94.

17. Zhou X, Yan DM, Zhu WF, Liu WJ, Nie HY, Xu S, et al.Efficacy and safety of Hou Gu Mi Xi in patients with spleen qi deficiency syndrome who underwent radical gastrectomy for gastric cancer: protocol for a multicenter, randomized, double-blind, placebo-controlled trial. Trials.2019; 20(1):343.

18. Okabe H, Kinjo Y, Obama K, Hosogi H, Hata H, Asao Y, et al.A Randomized Phase II Study of S-1 Adjuvant Chemotherapy With or Without Hochu-ekki-to, a Japanese Herbal Medicine, for Stage II/III Gastric Cancer: The KUGC07 (SHOT) Trial. Frontiers in oncology. 2019; 9:294.

19. Hung KF, Hsu CP, Chiang JH, Lin HJ, Kuo YT, Sun MF,et al.Complementary Chinese herbal medicine therapy improves survival of patients with gastric cancer in Taiwan: A nationwide retrospective matched-cohort study. Journal of ethnopharmacology.2017;199:168-74.

20. Lee YK, Bae K, Yoo HS, Cho SH.Benefit of Adjuvant Traditional Herbal Medicine With Chemotherapy for Resectable Gastric Cancer. Integr Cancer Ther .2018; 17(3):619-27. 
21. Xu Y, Zhao AG, Li ZY, Zhao G, Cai Y, Zhu XH,et al: Survival benefit of traditional Chinese herbal medicine (a herbal formula for invigorating spleen) for patients with advanced gastric cancer. Integr Cancer Ther. 2013;12(5):414-22.

22. Zhao L, Zhao AG, Zhao G, Xu Y, Zhu XH, Cao ND, et al.Survival benefit of traditional chinese herbal medicine (a herbal formula for invigorating spleen) in gastric cancer patients with peritoneal metastasis. Evidence-based complementary and alternative medicine : eCAM 2014;(2014):625493.

\section{Figures}

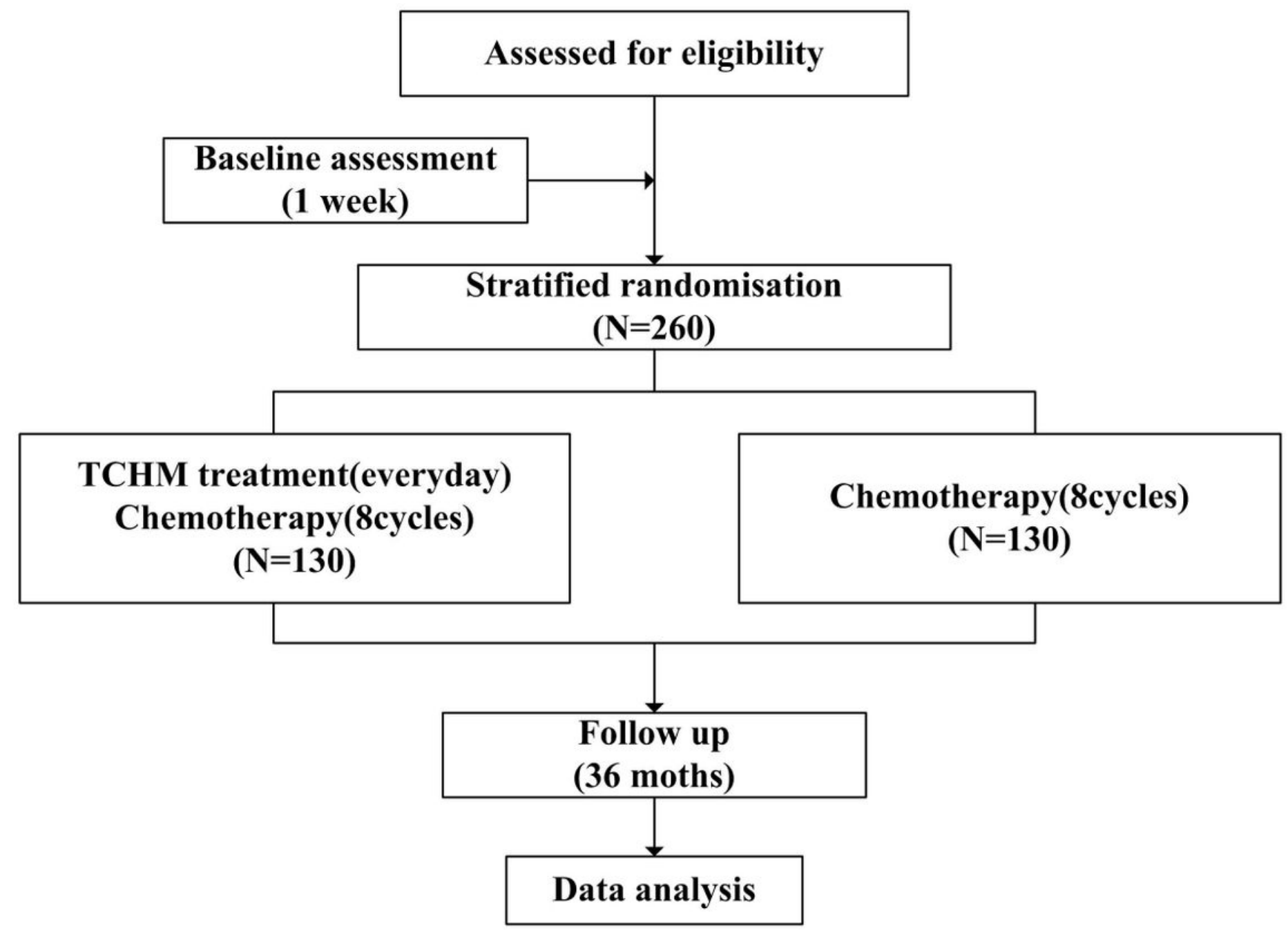

Figure 1

The trial design and checklist for this study protocol

\section{Supplementary Files}

This is a list of supplementary files associated with this preprint. Click to download.

- SPIRIT1.doc 